\title{
IROKAGE: A TOWNSCAPE COLOUR PLANNING SUPPORT SYSTEM USING KANSEI AND COLOUR HARMONY EVALUATIONS
}

\author{
Yuichiro KINOSHITA, Yoshiaki SAKAKURA, Eric W. COOPER and Katsuari KAMEI \\ Department of Human and Computer Intelligence, Ritsumeikan University \\ 1-1-1 Noji-higashi, Kusatsu, Shiga 525-8577, Japan
}

\begin{abstract}
Townscape colours have been a main factor in urban development. For townscape colours, keeping colour harmony within the environment is a common goal. In addition to the colour harmony, expressing characteristics and impressions of the town in townscape colours are other meaningful goals. This paper describes IroKage, a colour planning support system for improving townscapes. The system offers some colour combination proposals based on three elements: colour harmony, impressions of the townscape, and cost for the change of colours. First, two evaluation models to quantify colour harmony and impression of the townscape are developed from the approach of colour harmony principles and Kansei engineering, respectively. Next, the system is constructed using an evolutionary algorithm and the two evaluation models. After the construction, performance tests are conducted. The results show that the system has achieved sufficient ability to propose appropriate colour combinations with minimum colour changes.
\end{abstract}

Keywords: Kansei modelling, evaluation, townscape, colour harmony, evolutionary algorithm

\section{INTRODUCTION}

The problem of how to live in a comfortable environment has attracted attention with the changing expectation of residents, and the townscape is a central factor in urbandevelopment problems. Aesthetics of townscapes have been studied in various areas including the areas of environmental psychology and environmental engineering from various approaches $[1,2]$. The colour scheme of the buildings is one of the most influential factors concerning the townscape aesthetic. Several studies regarding townscape colours have been reported. In considering colours of buildings, each nearby colour in the row is a key point. The colours should be well matched and balanced. Proper evaluation of colours has to consider the whole street, not only individual buildings. There have been several colour studies reported as colour harmony principles. Using one of these principles may enable evaluation of the colours in the whole street and allow a better colour scheme for the townscape to be found.

However, characteristics of the target town also need to be respected throughout the colour planning process. In addition to form and material of the buildings in the town, climate and the historical background of the town are also elements affecting the characteristics, and these elements evoke particular impressions as well. Thus, expressing characteristics and impressions of a town in the townscape colours will be meaningful goals. Although several previous colour plans have included characteristics and impressions of towns, most of them have been based on anecdotal reports or experience and have been conducted for individual localities.

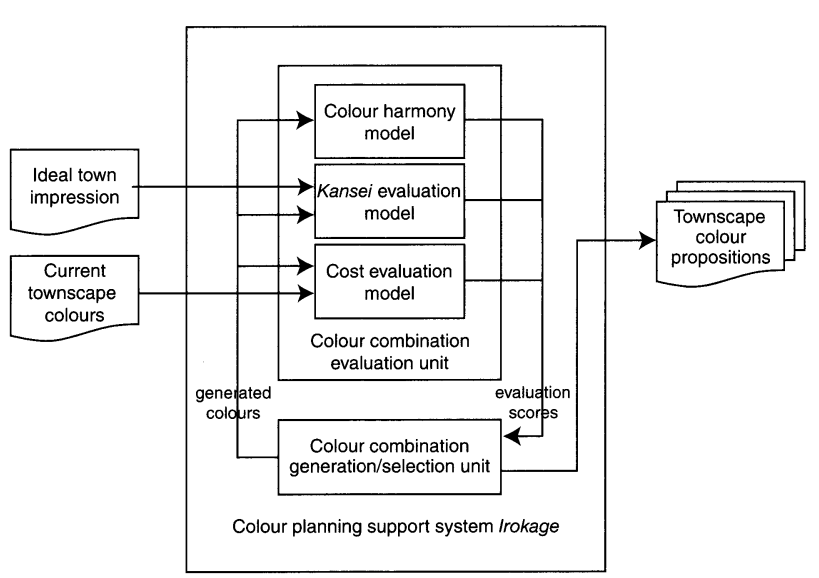

Figure 1: Block diagram of the colour planning support system.

This paper proposes IroKage, a colour planning support system for townscapes. The system finds the best colour co-ordination for townscape colours by considering the town impressions as well as colour harmony. Handling the impressions of the townscape and colour harmony requires quantification of psychological responses. In our previous studies, evaluation models to quantify the human responses by linking town impressions or colour harmony and townscape colours were constructed [3, 4]. Here, we discuss the implementation of the support system from the approach of an evolutionary algorithm [5] using the evaluation models.

\section{COLOUR PLANNING SUPPORT SYSTEM FOR TOWNSCAPE}

\subsection{Overview}

The inputs to the colour planning support system are the 
current townscape colours and ideal town impressions expressed with adjectives. The system offers several colour propositions for the townscape, as shown in Figure 1. In the system, the colour combination generation/selection unit generates colour combinations. The generated combinations are evaluated in the colour combination evaluation unit. The system outputs several high scoring colour combinations as the colour propositions.

\subsection{Colour combination evaluation unit}

The colour combination evaluation unit evaluates the colour combinations generated from the generation/selection unit one by one. First, the generated colour combination is evaluated from the approach of colour harmony. Quantification of colour harmony is required for the evaluation. Although various colour harmony studies have been reported previously, most studies are conceptually based and have no discussions about the quantification of colour harmony. Moon and Spencer arranged the ideas of colour harmony principles and quantified colour harmony as an aesthetic measure [6-8]. A colour harmony model for townscapes was constructed based on their colour harmony equations [3].

In addition to the colour harmony model, impressions of the generated colour combination are evaluated in terms of their suitability when compared with the ideal impressions of the townscape. The differences between the ideal impressions of the townscape and the psychological response for the generated colour combination are calculated. The combination having the smallest difference is evaluated as the appropriate combination. This process requires quantification of the response. The relationships between town impressions and townscape colours were modelled as a Kansei evaluation model [4].

The targets of this system are mostly townscapes that already exist. It is practically impossible to change all the colours of a townscape even if the best townscape design is found. In many cases, a portion of the colours may be changed. At this point, a critical issue is finding the most effective colour scheme changes for the lowest cost. The cost evaluation model calculates the cost to convert the current townscape colours to the generated colours.

\subsection{Colour combination generation/selection unit}

The colour combination generation/selection unit generates a large number of colour combinations. From the generated combinations, the unit selects several colour propositions for the output of the system based on scores obtained by the evaluation unit.

\section{COLOUR HARMONY MODEL}

\subsection{Aesthetic measure for colour harmony}

Moon and Spencer brought together the concepts in previous colour harmony principles as regions of harmony and ambiguity [6]. Every pair of colours is classified by region based on differences of hue, value, and chroma between the two colours in the Munsell colour space [9]. The general idea of an aesthetic measure $M$ is discussed by Birkhoff [10] and defined as

$$
M=\frac{O}{C}
$$

where $O$ represents the number of elements of order, and $C$ is the number of elements of complexity. Based on the regions of harmony and ambiguity, Moon and Spencer proposed equations for determining $O$ and $C$ in order to apply the measure to colour harmony [8].

\subsection{Fuzzy aesthetic measure for townscape colours}

The colour harmony equations by Moon and Spencer are intended for the use of general designs. In this study, we expanded their aesthetic measure for townscape colours and constructed the colour harmony model using Takagi-Sugeno type fuzzy reasoning [11]. The inputs to the model are townscape colours and the output is the aesthetic measure. At this time, only the colours of buildings in a townscape were considered and other elements such as plants etc. were excluded.

In the fuzzy aesthetic measure, the elements of order between two colours are calculated first. After that, the elements of order for a whole townscape $O_{\text {fuzzy }}$ is calculated by

$$
\begin{aligned}
O_{\text {fuzzy }}= & N_{\mathrm{g}}+\sum_{i=1}^{N_{\text {colour }}} \sum_{j=1}^{N_{\text {colour }}} o_{i j} \\
o_{i j}= & o_{i j}^{\text {hue }}+o_{i j}^{\text {value }}+o_{i j}^{\text {chroma }} \\
& (i \neq j)
\end{aligned}
$$

where $N_{\text {colour }}$ represents the number of colours and $N_{\mathrm{g}}$ represents the number of achromatic colour pairs. $o_{i j}$ represents the elements of order between colour $i$ and colour $j . o_{i j}^{\text {hue }}, o_{i j}^{\text {value }}$ and $o_{i j}^{\text {chroma }}$ represent the hue, value, and chroma elements of $o_{i j}$, respectively.

Based on Moon and Spencer's regions of harmony and ambiguity, membership functions are determined as shown in Figure 2. In the figure, $\Delta h u e_{i j}, \Delta v a l u e_{i j}$ and $\Delta c h r o m a_{i j}$ represent the hue difference, value difference and chroma difference between colour $i$ and colour $j$, respectively. $\mu_{\mathrm{v}}$ and $\mu_{\mathrm{c}}$ are calculated based on those membership functions under the constraint given by

$$
\mu_{\mathrm{v}}(r, \theta)=\mu_{\mathrm{c}}(r, \theta) \quad\left(\theta \neq 0, \frac{\pi}{2}, \pi, \frac{3}{2} \pi\right)
$$

where

$$
\Delta \text { value }_{i j}=r \sin \theta, \Delta \text { chroma }_{i j}=r \cos \theta .
$$




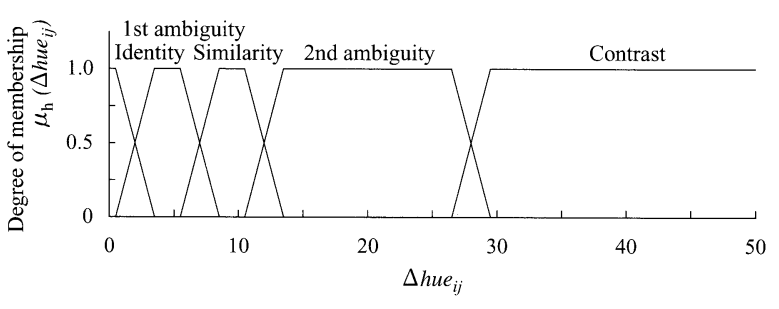

(a) hue

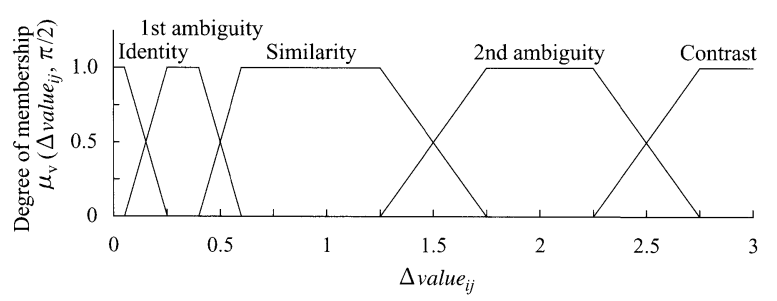

(b) value

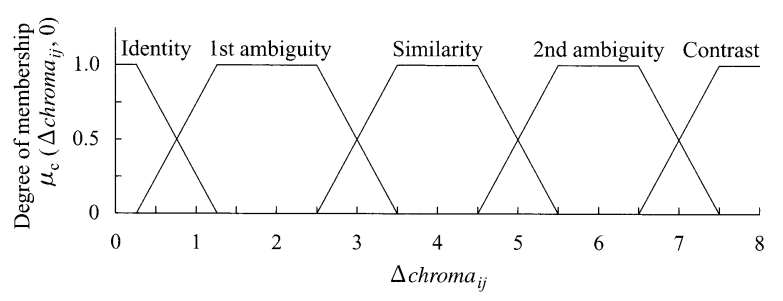

(c) chroma

Figure 2: Membership functions of the fuzzy subsets of identity, 1st ambiguity, similarity, 2nd ambiguity and contrast.

Table 1 shows fuzzy rules to calculate the elements of order between two colours.

In many Japanese cities, avoiding high chroma colours is suggested in townscape conservation regulations [12]. For the townscape colour harmony, high chroma colours will obstruct aesthetics. These elements are subtracted from aesthetic elements in the measure. Finally, the fuzzy aesthetic measure for townscape colours $M_{\text {fuzzy }}$ is calculated as

$$
\begin{aligned}
M_{\text {fuzzy }} & =\frac{O_{\text {fuzzy }}}{C}-\sum_{i=1}^{N_{\text {colour }}} u_{i} \\
C & =N_{\text {colour }}+N_{\mathrm{hDif}}+N_{\mathrm{vDif}}+N_{\mathrm{cDif}}
\end{aligned}
$$

where $N_{\mathrm{hDif}}$ represents the number of pairs of colours having noticeable hue difference. Likewise, $N_{\mathrm{vDif}}$ represents the number of pairs with value difference, and $N_{\text {cDif }}$ is for chroma difference. $u_{i}$ represents the degree of aesthetic obstruction for colour $i$. Figure 3 shows the membership function of chroma values. Table 2 shows the fuzzy rules to calculate the aesthetic obstruction.

\subsection{Evaluation of colour harmony model}

Computer-based model evaluation was conducted using the semantic differential (SD) method [13] to make sure that the output from the colour harmony model is proper. For the evaluation, pictures of a residential area are used as samples. Fifty coloured picture samples were made by
Table 1: Fuzzy rules for calculation of elements of order.

\begin{tabular}{|l|l|}
\hline \multicolumn{1}{|c|}{ antecedent part } & \multicolumn{1}{c|}{ consequent part } \\
\hline$\Delta h u e_{i j}$ is Identity & $o_{i j}^{\text {hue }}$ is 1.5 \\
\hline$\Delta h u e_{i j} 1$ st ambiguity & $o_{i j}^{\text {hue }}$ is 0.0 \\
\hline$\Delta h u e_{i j}$ is Similarity & $o_{i j}^{\text {hie }}$ is 1.1 \\
\hline$\Delta h u e_{i j}$ is 2 nd ambiguity & $o_{i j}^{\text {hie }}$ is 0.65 \\
\hline$\Delta h u e_{i j}$ is Contrast & $o_{i j}^{\text {hiu }}$ is 1.7 \\
\hline$\Delta v a l u e_{i j}$ is Identity & $o_{i j}^{\text {value }}$ is -1.3 \\
\hline$\Delta v a l u e_{i j}$ is 1 st ambiguity & $o_{i j}^{\text {value }}$ is -1.0 \\
\hline$\Delta v a l u e_{i j}$ is Similarity & $o_{i j}^{\text {value }}$ is 0.7 \\
\hline$\Delta v a l u e_{i j}$ is 2nd ambiguity & $o_{i j}^{\text {value }}$ is -0.2 \\
\hline$\Delta v a l u e_{i j}$ is Contrast & $o_{i j}^{\text {value }}$ is 3.7 \\
\hline$\Delta$ chroma $_{i j}$ is Identity & $o_{i j}^{\text {chroma }}$ is 0.8 \\
\hline$\Delta$ chroma $_{i j}$ is 1 st ambiguity & $o_{i j}^{\text {chroma }}$ is 0.0 \\
\hline$\Delta$ chroma $_{i j}$ is Similarity & $o_{i j}^{\text {chroma }}$ is 0.1 \\
\hline$\Delta$ chroma $_{i j}$ is 2nd ambiguity & $o_{i j}^{\text {chroma }}$ is 0.0 \\
\hline$\Delta$ chroma $_{i j}$ is Contrast & $o_{i j}^{\text {chroma }}$ is 0.4 \\
\hline
\end{tabular}

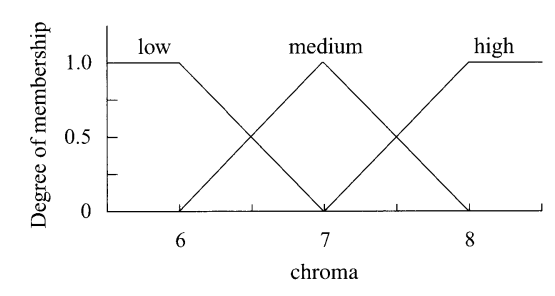

Figure 3: Membership function of chroma values.

altering the same picture while maintaining all other conditions. Adjectives were used to evaluate the colour harmony of the samples. The meaning of harmonious is ambiguous and there are many definitions. In this study, we defined the three adjectives shown in Table 3 as subsets of the fuzzy set harmonious. The subjects evaluate the samples using the three adjectives instead of the term of harmonious. One picture sample and a five-step SD scale are placed on a computer screen. The subjects choose a suitable response on the scale. The subjects evaluate all of 50 picture samples with three pairs of adjectives. The samples are shown at random to reduce influence from the order of the presentation.

\subsection{Results}

The experiments were conducted for 20 subjects. To clarify the relationship between the three adjective pairs, factor analysis was conducted based on the experiment results for the 50 samples. Table 4 shows the result of the factor analysis. Only one factor was extracted and the contribution ratio of the factor is over $96 \%$. Also, every factor loading is over 0.97 . This indicates that the three adjectives have almost the same meaning. Therefore, the average of the scores of the three adjective pairs are simply adopted as evaluation of colour harmony. The scores from the subjects were compared with the output values from 
the colour harmony model, as shown in Figure 4. The accuracy of the output was sufficient, in that the correlation coefficient for subject response and model output was $r=0.57$. The result of a significance test showed the correlation was significant at the $1 \%$ level.

\section{KANSEI EVALUATION MODEL}

\subsection{Evaluation experiment of town impressions}

A Kansei evaluation model is constructed using the Kansei engineering approach. This technique translates target psychological responses into perceptual design elements [14, 15]. First, evaluation experiments were conducted to investigate the relationship between town

Table 2: Fuzzy rules for calculation of aesthetic obstruction.

\begin{tabular}{|l|l|}
\hline antecedent part & consequent part \\
\hline chroma is low & $u_{i}$ is 0 \\
\hline chroma is medium & $u_{i}$ is $0.25 \times$ chroma -1.5 \\
\hline chroma is high & $u_{i}$ is 0.5 \\
\hline
\end{tabular}

Table 3: Pairs of adjectives related to colour harmony.

\begin{tabular}{|c|c|}
\hline adjective No. & adjectives \\
\hline 1 & strange - not strange \\
\hline 2 & disorderly - orderly \\
\hline 3 & displeasing - pleasing \\
\hline
\end{tabular}

Table 4: Result of the factor analysis.

\begin{tabular}{|l|c|}
\hline variable & factor 1 \\
\hline strange-not strange & 0.988 \\
\hline disorderly-orderly & 0.985 \\
\hline displeasing-pleasing & 0.979 \\
\hline contribution ratio (\%) & 96.798 \\
\hline
\end{tabular}

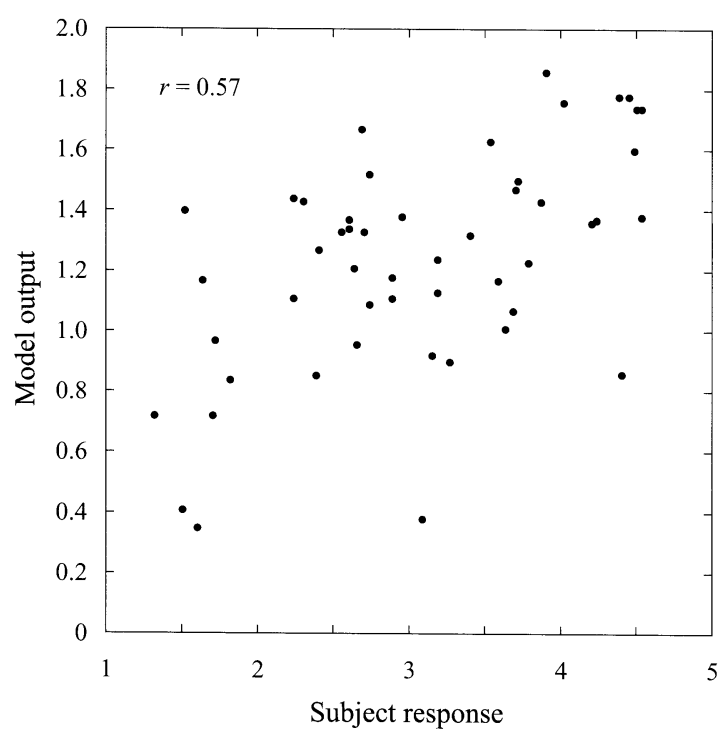

Figure 4: The comparison between the result of the evaluation and the output from the colour harmony model. impressions and townscape colours. Adjectives allow expression of the psychological responses. In this study, adjectives express the impressions of the townscape. First, approximately 470 adjectives related to town impressions were collected from dictionaries and previous studies. After collection, similar adjectives were combined and they were paired with opposite meanings. Finally, the number of adjectives was reduced to sixteen pairs.

One-hundred colour picture samples were prepared for the evaluation experiments. The pictures show the front side of a row of houses. These were the same configuration as the samples for the colour harmony evaluation but colours in the samples are different.

Computer-based evaluation experiments were conducted for 20 subjects, four females and sixteen males, using the SD method. One picture sample and the sixteen adjective pairs are placed on the computer screen. In the experiments, the subjects select a suitable response on the SD scales $\{1,2, \ldots, 5\}$ for each pair of adjectives. The subjects evaluated all of the 100 picture samples, which are shown at random.

\subsection{Selection of adjectives}

After the experiments, appropriate adjectives for the model construction were selected based on the results of the above experiments. An evaluation in the centre of the SD scale implies that the adjective was evaluated as neutral. The adjectives with many neutral evaluations may be irrelevant for expressing town impressions. Four adjectives were excluded in this process. Variance of evaluation scores is also a factor to be considered. We checked the variance for each sample but every pair of adjectives showed low variance. No adjective was excluded in this process. Ultimately, twelve adjectives were selected to construct the Kansei model, as shown in Table 5.

\subsection{Construction of Kansei evaluation model}

The behaviour of Kansei response is usually non-linear

Table 5: Pairs of adjectives related to townscape impressions.

\begin{tabular}{|c|r|}
\hline adjective No. & adjectives \\
\hline 1 & cold - warm \\
\hline 2 & unrefined - refined \\
\hline 3 & restless - calm \\
\hline 4 & unfriendly - friendly \\
\hline 5 & uncomfortable - comfortable \\
\hline 6 & artificial - natural \\
\hline 7 & typical - individualistic \\
\hline 8 & conservative - progressive \\
\hline 9 & quiet - lively \\
\hline 10 & old-fashioned - modern \\
\hline 11 & awkward - elegant \\
\hline 12 & western - eastern \\
\hline
\end{tabular}




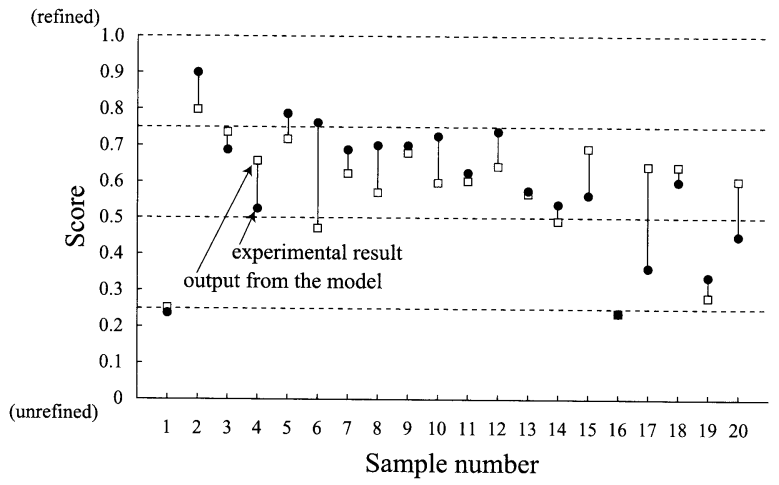

(a) adjective No. 2 unrefined - refined

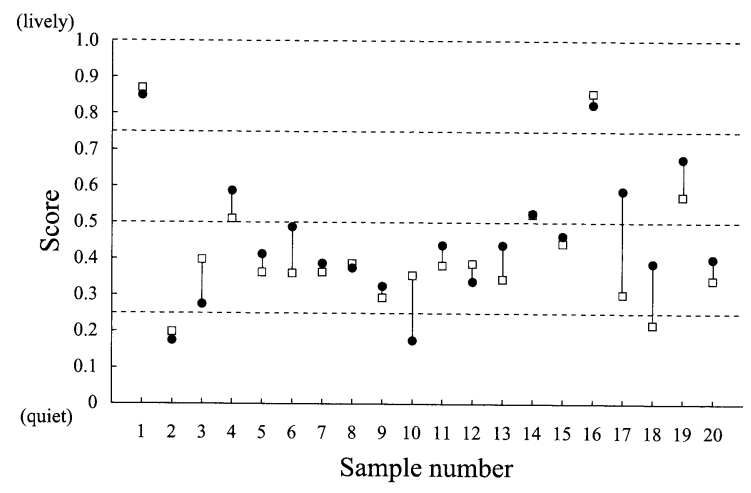

(b) adjective No. 9 quiet - lively

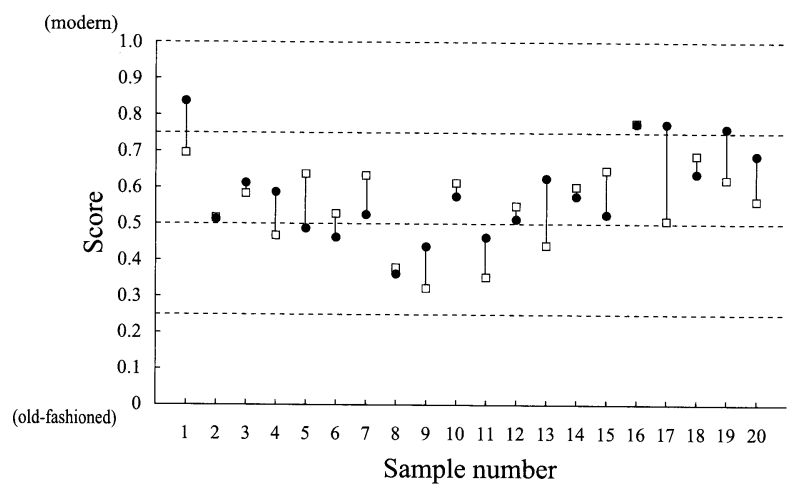

(c) adjective No. 10 old-fashioned - modern

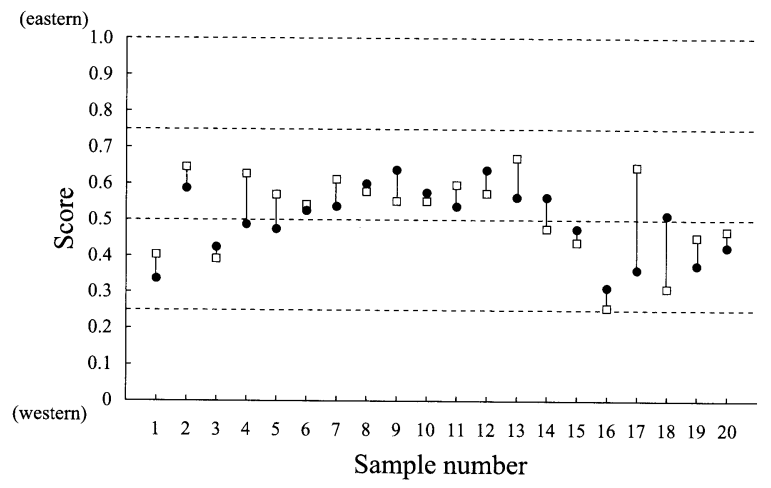

(d) adjective No. 12 western - eastern

Figure 5: Examples of the testing results. and it is difficult to manage Kansei data using conventional methods. Some previous studies adopted neural networks for Kansei data [16, 17]. Neural networks are one of the best methods for modelling non-linear data. We constructed a model using a neural network for each pair of adjectives. Sixty-two experimental results were selected for the training data of the neural networks and 20 results were selected for the testing data. Both training and testing data were selected to consist of various, dissimilar colour combinations. Each colour in the data was selected to have unique hue, brightness or saturation attributes. The input items to each neural network are the wall colours, roof colours, and window frame colours of the houses in the picture samples. Every colour is expressed in the CIELAB colour system [9]. The three CIELAB values, $L^{*}, a^{*}$ and $b^{*}$, are used for the wall colours and roof colours, and only $L^{*}$ is used for the window frame colours because the use of chromatic colours is limited. Since there are three buildings in the sample picture, the input data becomes 21 values. The output from each neural network is the SD scale response for the town impression. Every value is given in $[0,1]$. Each neural network has three layers and the numbers of units are 21,30 , and 1 on the input, hidden, and output layers, respectively. Every network was trained using back-propagation learning to an accuracy of $3.125 \times 10^{-4}$ mean square error, which is equivalent to 0.1 on the SD scale.

\subsection{Results}

After the model construction, a performance test was conducted. We input the 20 testing data into the constructed model and calculated errors between the output from the model and the average of the experimental results. Examples of the testing results are shown in Figure 5. Scores $0.0,0.25,0.5,0.75$, and 1.0 shown by the broken lines in the figures correspond to the SD scales $\{1,2, \ldots$, $5\}$. Testing of the model shows that the error was within 0.25 for over $90 \%$ of the samples. This result demonstrates that our Kansei model has adequate ability to evaluate the town impressions.

\section{COLOUR COMBINATION GENERATION/ SELECTION UNIT}

\subsection{Implementation approach}

The colour combination generation/selection unit generates a large number of colour combinations based on current townscape colours input to the support system. The unit also selects some colour combinations as the system output according to the evaluation values from the three models: the colour harmony model, Kansei evaluation model and cost evaluation model. 
Here, the problem is the method to select appropriate combinations under the evaluation scores with different dimensions. In our previous study, we conducted experiments to investigate methods of applying human sensibilities to the selection of samples with different dimensions. The results showed that one outstanding evaluation score has a big impact on the final selection and other evaluation scores do not affect it so strongly [18]. In this case, the average of the three evaluation scores does not work for the selection and outstanding scores need to be treated individually. For selecting appropriate colour schemes to output from the system, we focuse on Pareto optimal solutions.

In a maximization problem, $a$ is said to dominate $b$ when

$$
f_{e}(a) \geq f_{e}(b)(\forall e \in\{1,2, \ldots, n\}, a \neq b)
$$

where $f_{e}$ represents a vector function, and $a$ and $b$ represent two decision vectors. A decision vector which is not dominated by any other vector is said that it is nondominated. The decision vectors that are nondominated within the entire search space are called Pareto optimal solutions. We search for those solutions from an evolutionary algorithm approach [5].

\subsection{Encoding}

The system uses wall, roof and window frame colours of three buildings, nine colours in total. In the evolutionary algorithm, the colours are expressed in the CIELAB colour system and encoded by value encoding. Each wall colour and roof colour consists of the three CIELAB values, $L^{*}, a^{*}$ and $b^{*}$. Each window frame colour consists of $L^{*}$ value only. Seven values therefore express the colour scheme of one building. In total, one individual includes 21 values, as shown in Figure 6. Every $L^{*}$ value is given in $[0,100]$ while $a^{*}$ and $b^{*}$ values are given in $[-50,50]$.

\subsection{Colour search algorithm}

\subsubsection{Overview}

The generation/selection unit selects townscape colour propositions using an evolutionary algorithm. Figure 7 shows the colour search procedure in the system. First, the population of the first generation individuals is generated. The individuals are evaluated based on the three models. According to the evaluation scores, individuals are

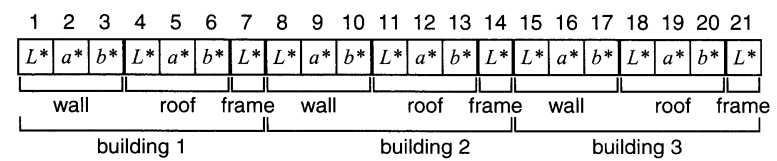

Figure 6: Encoding of individuals. selected as the parents of the next generation. After that, crossover and mutations are executed for the selected individuals at a given probability. Finally, those individuals will be the population of the next generation. The system stores all individuals through all generations and selects the highest ranking individuals for the output.

\subsubsection{Initialization}

In this stage, the population of the first generation is generated by altering several colours in the current townscape input. First, up to three colours are chosen as targets for alteration. Next, one of the three alteration patterns shown in Figure 8 is applied to each target colour randomly. In pattern (a), all $L^{*} a^{*} b^{*}$ values of the target colour are replaced with random numbers. The remaining two patterns change only one or two $L^{*} a^{*} b^{*}$ value(s). In pattern (b), the $L^{*}$ value of the target is replaced with random number. This means only the lightness attribute of the target is altered and the other attributes are kept the same. Likewise, the $a^{*}$ and $b^{*}$ values are replaced in pattern (c). The hue and saturation attributes are altered and the lightness is kept the same in this pattern. When a window frame colour is chosen as the alternation target, the $L^{*}$ value is replaced with either ' 10 ' (almost black) or '90' (almost white) randomly.

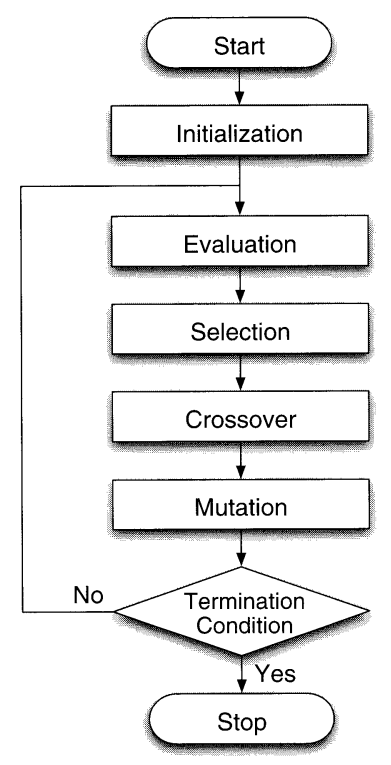

Figure 7: Colour search procedure.
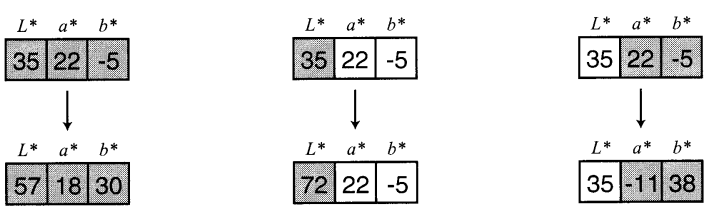

(a) all of $L^{*}, a^{*}$ and $b^{*}$

(b) $L^{*}$ only

(c) $a^{*}$ and $b^{*}$ only

Figure 8: Colour alteration patterns in the initialization and mutation processes. 
$\begin{array}{lllllllllllllllllllll}1 & 2 & 3 & 4 & 5 & 6 & 7 & 8 & 9 & 10 & 11 & 12 & 13 & 14 & 15 & 16 & 17 & 18 & 19 & 20 & 21\end{array}$ \begin{tabular}{|l|l|l|l|l|l|l|l|l|l|l|l|l|l|l|l|l|l|l|l|l|}
\hline$L^{*}$ & $a^{*}$ & $b^{*}$ & $L^{*}$ & $a^{*}$ & $b^{*}$ & $L^{*}$ & $L^{*}$ & $a^{*}$ & $b^{*}$ & $L^{*}$ & $a^{*}$ & $b^{*}$ & $L^{*}$ & $L^{*}$ & $a^{*}$ & $b^{*}$ & $L^{*}$ & $a^{*}$ & $b^{*}$ & $L^{*}$ \\
\hline
\end{tabular}

Figure 9: Candidate crossover points.

Table 6: Test sample colours.

\begin{tabular}{|c|c|c|c|c|c|c|c|c|}
\hline \multirow{2}{*}{$\begin{array}{c}\text { sample } \\
\text { No. }\end{array}$} & \multirow{2}{*}{$\begin{array}{c}\text { building } \\
\text { No. }\end{array}$} & \multicolumn{3}{|c|}{ wall } & \multicolumn{3}{|c|}{ roof } & \multirow{2}{*}{$\begin{array}{c}\text { frame } \\
L^{*} \\
\end{array}$} \\
\hline & & $L^{*}$ & $a^{*}$ & $b^{*}$ & $L^{*}$ & $a^{*}$ & $b^{*}$ & \\
\hline \multirow{3}{*}{1} & 1 & 35 & 20 & -20 & 45 & 0 & 0 & 90 \\
\hline & 2 & 55 & 0 & 0 & 45 & 0 & 0 & 90 \\
\hline & 3 & 35 & -20 & -20 & 45 & 0 & 0 & 90 \\
\hline \multirow{3}{*}{2} & 1 & 80 & 0 & -10 & 45 & 0 & 0 & 10 \\
\hline & 2 & 55 & 40 & 20 & 45 & 0 & 0 & 90 \\
\hline & 3 & 80 & 0 & 15 & 40 & 20 & 30 & 10 \\
\hline \multirow{3}{*}{3} & 1 & 80 & 0 & 0 & 45 & 0 & 0 & 90 \\
\hline & 2 & 90 & 0 & 10 & 45 & 0 & 0 & 90 \\
\hline & 3 & 80 & 0 & 0 & 45 & 0 & 0 & 90 \\
\hline
\end{tabular}

Table 7: Parameters for the evolutionary algorithm.

\begin{tabular}{|c|c|}
\hline population size & 100 \\
\hline crossover probability & 0.9 \\
\hline mutation probability & 0.03 \\
\hline number of generations & 300 \\
\hline
\end{tabular}

\subsubsection{Evaluation}

This stage evaluates the individuals using the three evaluation models. For the colour harmony evaluation, all $L^{*} a^{*} b^{*}$ values in an individual are transformed to Munsell values. The combination of Munsell values is evaluated in the colour harmony model. For the evaluation of townscape impressions, $L^{*} a^{*} b^{*}$ values in an individual are directly input to the Kansei model. The model outputs the fitness based on the ideal impressions input to the support system. In terms of the colour changing cost, the repaint cost is assumed to be almost the same even if the new colour is similar to the original colour. The number of colour changes is simply applied for the cost evaluation.

\subsubsection{Selection}

This stage first calculates the rank of each individual under the evaluation scores. The rank of individual $i$ is given by

$$
r_{i}=N_{\mathrm{p}}-n_{i}
$$

where $N_{\mathrm{p}}$ represents the number of populations, and $n_{i}$ represents the number of individuals dominating individual $i$. The individual $i$ is selected as a parent by the selection probability $P(i)$ given by

$$
P(i)=\frac{r_{i}}{\sum_{j=1}^{N_{\mathrm{p}}} r_{j}}
$$

\subsubsection{Crossover}

Single point crossover is applied to the selected parents. The crossover is randomly executed at one of the eight crossover points shown in Figure 9, and is not executed between $L^{*}$ and $a^{*}$, or between $a^{*}$ and $b^{*}$. The values after the crossover point swap between one parent and another parent. This stage shuffles combinations of colours but does not modify colours themselves.

\subsubsection{Mutation}

Each of nine colours in an individual mutates with a certain probability. For wall colours and roof colours, one of the three mutation patterns as shown in Figure 8 is applied randomly. In the same way as the initialization process, the mutation target colour is replaced with a random colour in pattern (a). In pattern (b), only the lightness attribute of the target is altered. In pattern (c), the hue and saturation attributes are altered and the lightness attribute is kept the same. When the mutation is executed at a window frame colour, the lightness value $L^{*}$ is replaced with either ' 10 ' or ' 90 ' at random.

\section{RESULTS AND DISCUSSION}

We tested the performance of our colour planning support system using the test samples shown in Table 6 . The parameters for the evolutionary algorithm are shown in Table 7. At this time, we set warm and calm as the ideal impressions for sample No. 1, refined and natural for sample No. 2, and elegant for sample No. 3.

Table 8 shows the examples of the system output for sample No. 1. The bold face numbers indicate modified colours. Figure 10 shows repaint simulations with the output colours. In the figure, the numbers in parentheses indicate the colour harmony and Kansei evaluation scores, respectively. The initial evaluation scores of the test sample No. 1 are 1.35 by the colour harmony model and 0.12 by the Kansei evaluation model. As shown in the examples, the system mainly output the combination of warm colours, yellowish and reddish colours. These match to the ideal impression warm. The wall colour of the right building, viridian, was changed in every case. This means the viridian colour evokes cold and/or restless impression. Result No. 1A has a red brown colour instead of the viridian and it achieved 0.54 Kanse $i$ score only with the one colour change. Result No. 1B, with two colour changes, achieved 0.66 Kansei score and also increased the colour harmony score. We got some other colour combinations with higher evaluations in terms of colour harmony and Kansei, but cost evaluation was not sufficient in those cases.

Table 9 shows the examples of the system output for sample No. 2, and Figure 11 shows their repaint simula- 
Table 8: System output examples for test sample No. 1.

\begin{tabular}{|c|c|c|c|c|c|c|c|c|}
\hline \multirow{2}{*}{$\begin{array}{c}\text { result } \\
\text { No. }\end{array}$} & building & \multicolumn{3}{|c|}{ wall } & \multicolumn{3}{|c|}{ roof } & frame \\
\cline { 2 - 9 } & No. & $L^{*}$ & $a^{*}$ & $b^{*}$ & $L^{*}$ & $a^{*}$ & $b^{*}$ & $L^{*}$ \\
\hline \multirow{4}{*}{ 1A } & 1 & 35 & 20 & -20 & 45 & 0 & 0 & 90 \\
\cline { 2 - 10 } & 2 & 55 & 0 & 0 & 45 & 0 & 0 & 90 \\
\cline { 2 - 10 } & 3 & $\mathbf{2 6}$ & $\mathbf{3 4}$ & $\mathbf{3 6}$ & 45 & 0 & 0 & 90 \\
\hline \multirow{4}{*}{ 1B } & 1 & $\mathbf{6 7}$ & $\mathbf{3}$ & $\mathbf{3 8}$ & 45 & 0 & 0 & 90 \\
\cline { 2 - 10 } & 2 & 55 & 0 & 0 & 45 & 0 & 0 & 90 \\
\cline { 2 - 10 } & 3 & $\mathbf{7 0}$ & $\mathbf{6}$ & $\mathbf{7}$ & 45 & 0 & 0 & 90 \\
\hline \multirow{4}{*}{$1 \mathrm{C}$} & 1 & $\mathbf{8 1}$ & $\mathbf{- 3}$ & $\mathbf{1 1}$ & 45 & 0 & 0 & 90 \\
\cline { 2 - 9 } & 2 & 55 & 0 & 0 & 45 & 0 & 0 & 90 \\
\cline { 2 - 9 } & 3 & $\mathbf{7 7}$ & $\mathbf{2 7}$ & $\mathbf{4}$ & 45 & 0 & 0 & 90 \\
\hline
\end{tabular}

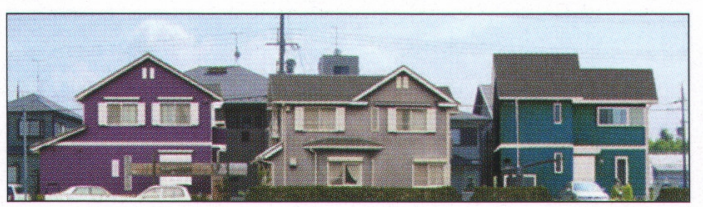

(a) original sample No. $1(1.35,0.12)$

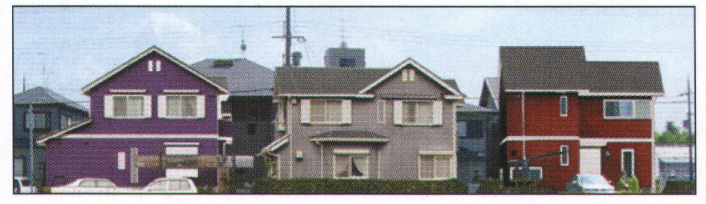

(b) result No. $1 \mathrm{~A}(1.07,0.54)$

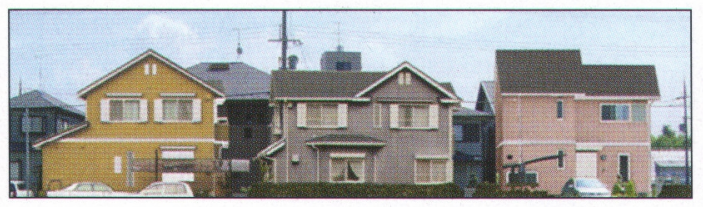

(c) result No. 1B $(1.09,0.66)$

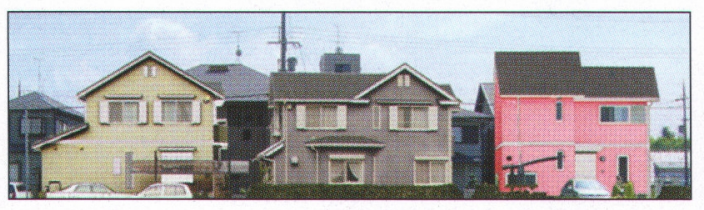

(d) result No. $1 \mathrm{C}(1.36,0.59)$

Figure 10: Repaint simulations for test sample No. 1.

tions. Sample No. 2 is initially evaluated as 1.11 by the colour harmony model and 0.39 by the Kansei model. For this sample, the variety of the system output included greenish, brownish and yellowish colours. In result No. $2 \mathrm{~A}$, the wall colour of the centre building has changed to a somewhat darker colour. With only a small change, the colour harmony score increased to 1.47 while the Kansei score had a little change at 0.42 . Result No. $2 B$, with three colour changes, also achieved not only a high colour harmony score, 1.52, but also a high Kansei score, 0.69. In most results, the colours of the right building were not changed. This means those colours are harmonious as well as fit for the ideal impressions. The system successfully modified only inappropriate colours and maintained
Table 9: System output examples for test sample No. 2.

\begin{tabular}{|c|c|c|c|c|c|c|c|c|}
\hline \multirow{2}{*}{$\begin{array}{c}\text { result } \\
\text { No. }\end{array}$} & building & \multicolumn{3}{|c|}{ wall } & \multicolumn{3}{|c|}{ roof } & frame \\
\cline { 2 - 10 } & $L^{*}$ & $a^{*}$ & $b^{*}$ & $L^{*}$ & $a^{*}$ & $b^{*}$ & $L^{*}$ \\
\hline \multirow{4}{*}{$2 \mathrm{~A}$} & 1 & 80 & 0 & -10 & 45 & 0 & 0 & 10 \\
\cline { 2 - 10 } & 2 & $\mathbf{4 9}$ & $\mathbf{2 5}$ & $\mathbf{- 5}$ & 45 & 0 & 0 & 90 \\
\cline { 2 - 10 } & 3 & 80 & 0 & 15 & 40 & 20 & 30 & 10 \\
\hline \multirow{3}{*}{$2 \mathrm{~B}$} & 1 & $\mathbf{5 3}$ & $\mathbf{- 7}$ & $\mathbf{2 6}$ & $\mathbf{2}$ & $\mathbf{- 1}$ & $\mathbf{- 7}$ & 10 \\
\cline { 2 - 10 } & 2 & $\mathbf{5 1}$ & $\mathbf{- 1 3}$ & $\mathbf{3 4}$ & 45 & 0 & 0 & 90 \\
\cline { 2 - 10 } & 3 & 80 & 0 & 15 & 40 & 20 & 30 & 10 \\
\hline \multirow{4}{*}{$2 \mathrm{C}$} & 1 & $\mathbf{4 8}$ & $\mathbf{1}$ & $\mathbf{1 5}$ & 45 & 0 & 0 & 10 \\
\cline { 2 - 9 } & 2 & 55 & 40 & 20 & 45 & 0 & 0 & $\mathbf{1 0}$ \\
\cline { 2 - 9 } & 3 & 80 & 0 & 15 & 40 & 20 & 30 & 10 \\
\hline
\end{tabular}

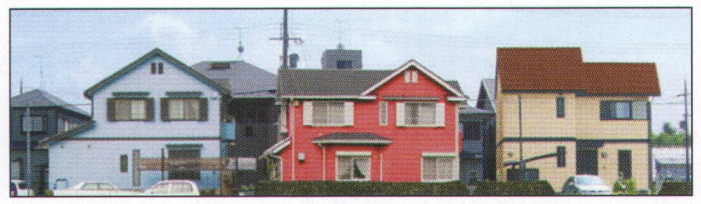

(a) original sample No. $2(1.11,0.39)$

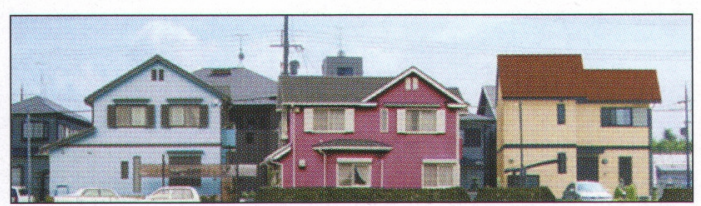

(b) result No. $2 \mathrm{~A}(1.47,0.42)$

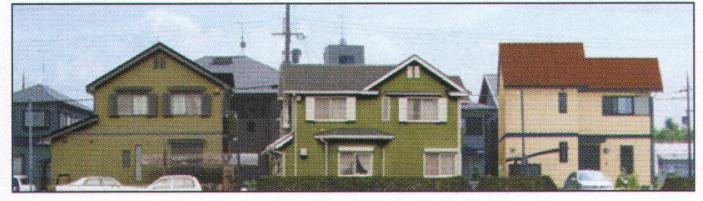

(c) result No. 2B $(1.52,0.69)$

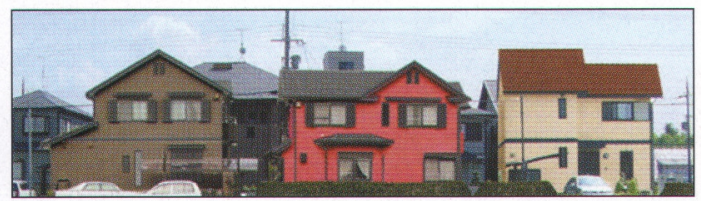

(d) result No. $2 \mathrm{C}(0.96,0.64)$

Figure 11: Repaint simulations for test sample No. 2.

good colour schemes.

Table 10 and Figure 12 show the examples of the system output for sample No. 3 and their repaint simulations, respectively. Sample No. 3 consists of white and grey colours only. The initial evaluation scores of sample No. 3 are 1.23 by the colour harmony model and 0.41 by the Kansei model. For this sample, the system proposed several dark colours to replace the original colours. In result No. 3A, the wall colour of the left building has been changed to dark purple. With this one colour change, the colour harmony score increased to 1.44 , while the Kansei score was almost the same, 0.44. Result No. 3B, with two colour changes, achieved 1.81 colour harmony score and 0.58 Kansei score. The colours of result No. 3B also 
Table 10: System output examples for test sample No. 3.

\begin{tabular}{|c|c|c|c|c|c|c|c|c|}
\hline \multirow{2}{*}{$\begin{array}{c}\text { result } \\
\text { No. }\end{array}$} & building & \multicolumn{3}{|c|}{ wall } & \multicolumn{3}{|c|}{ roof } & frame \\
\cline { 2 - 9 } & No. & $L^{*}$ & $a^{*}$ & $b^{*}$ & $L^{*}$ & $a^{*}$ & $b^{*}$ & $L^{*}$ \\
\hline \multirow{3}{*}{$3 \mathrm{~A}$} & 1 & $\mathbf{2 6}$ & $\mathbf{2 7}$ & $\mathbf{- 4}$ & 45 & 0 & 0 & 90 \\
\cline { 2 - 10 } & 2 & 80 & 0 & 0 & 45 & 0 & 0 & 90 \\
\cline { 2 - 9 } & 3 & 80 & 0 & 0 & 45 & 0 & 0 & 90 \\
\hline \multirow{4}{*}{$3 \mathrm{~B}$} & 1 & $\mathbf{3 8}$ & 0 & 0 & 45 & 0 & 0 & 90 \\
\cline { 2 - 9 } & 2 & $\mathbf{1 4}$ & 0 & 10 & 45 & 0 & 0 & 90 \\
\cline { 2 - 9 } & 3 & 80 & 0 & 0 & 45 & 0 & 0 & 90 \\
\hline \multirow{3}{*}{$3 \mathrm{C}$} & 1 & $\mathbf{4 0}$ & $\mathbf{1}$ & $\mathbf{1 6}$ & 45 & 0 & 0 & 90 \\
\cline { 2 - 9 } & 2 & $\mathbf{9}$ & $\mathbf{- 6}$ & $\mathbf{- 4}$ & 45 & 0 & 0 & 90 \\
\cline { 2 - 9 } & 3 & 80 & 0 & 0 & $\mathbf{5 2}$ & 20 & 30 & 90 \\
\hline
\end{tabular}

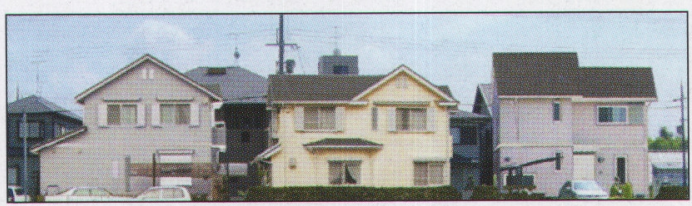

(a) original sample No. $3(1.23,0.41)$

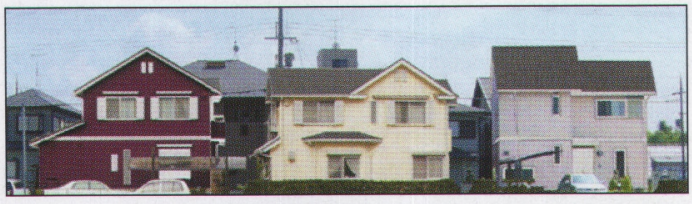

(b) result No. $3 \mathrm{~A}(1.44,0.44)$

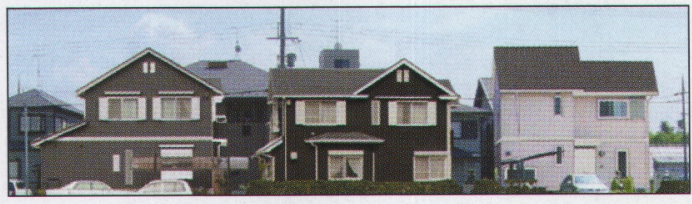

(c) result No. 3B $(1.81,0.58)$

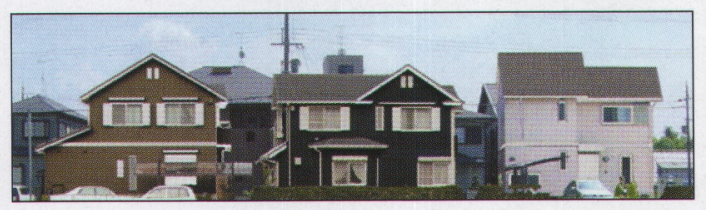

(d) result No. $3 \mathrm{C}(1.75,0.61)$

Figure 12: Repaint simulations for test sample No. 3.

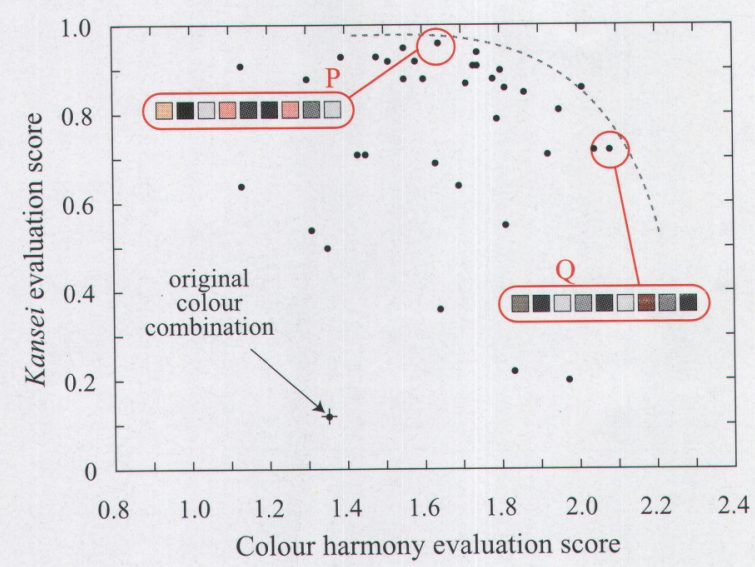

Figure 13: Typical individual distribution for test sample No. 1 as Pareto optimal solutions. include dark grey and dark brown. Those colours were generated by altering only the lightness attribute of the original colours.

Figure 13 shows the typical distribution of the output individuals as Pareto optimal solutions. These individuals are plotted for the colour harmony and Kansei evaluation axes. The axis of the cost evaluation is not shown here. For the final output, some different types of individuals were selected. The individual $\mathrm{P}$, which is located on the Pareto front, has an outstanding Kansei score. In the case of sample No. 1, these kinds of individuals consist of many warm colours, such as red and yellow. The individual Q, also on the Pareto front, is outstanding under the colour harmony score. These kinds of individuals commonly consist of many achromatic colours. These results indicate that the system proposed appropriate colour combinations and also a wide variety of combinations.

\section{CONCLUSIONS}

In this paper, we first proposed a colour planning support system for townscapes. The system offers colour combination proposals according to colour harmony, suitability for ideal town impressions and the cost to modify colours. The system was constructed using an evolutionary algorithm with Kansei and colour harmony evaluations. Testing of the system demonstrated that it has sufficient ability to propose appropriate colour combinations for the ideal impressions with minimum colour changes. In future studies, we are going to conduct colour planning in realworld townscapes by applying our system.

\section{REFERENCES}

1. Lynch, K.; The Image of the City; MIT Press, Cambridge, MA (1960).

2. Nasar, J. L.; The Evaluative Image of the City; Sage Publications, Thousand Oaks, CA (1998).

3. Kinoshita, Y., Cooper, E. W., Hoshino, Y. and Kamei, K.; A townscape evaluation system based on Kansei and colour harmony models, Proc. 2004 IEEE Int. Conf. Syst., Man, Cybern.; pp. 327-332 (2004).

4. Kinoshita, Y., Cooper, E. W. and Kamei, K.; A colour support system for townscape based on Kansei and colour harmony models, Proc. 4th Int. Symp. Advanced Intelligent Systems; pp. 51-54 (2003).

5. Coello Coello, C. A.; An updated survey of GA-based multiobjective optimization techniques, ACM Comput. Surv.; Vol. 32, No. 2, pp. 109-143 (2000).

6. Moon, P. and Spencer, D. E.; Geometric formulation of classical color harmony, J. Opt. Soc. Am.; Vol. 34, No. 1, pp. 46-59 (1944). 
7. Moon, P. and Spencer, D. E.; Area in color harmony, J. Opt. Soc. Am.; Vol. 34, No. 2, pp. 93-103 (1944).

8. Moon, P. and Spencer, D. E.; Aesthetic measure applied to color harmony, J. Opt. Soc. Am.; Vol. 34, No. 4, pp. 234-242 (1944).

9. Agoston, G. A.; Color Theory and Its Application in Art and Design; Springer-Verlag, Berlin (1987).

10. Birkhoff, G. D.; Aesthetic Measure; Harvard University Press, Cambridge, MA (1933).

11. Takagi, T. and Sugeno, M.; Fuzzy identification of systems and its applications to modeling and control, IEEE Trans. Syst., Man, Cybern.; Vol. SMC-15, No. 1, pp. 116-132 (1985).

12. For example, Yokosuka City; Townscape Conservation Regulation, http://www.city.yokosuka.kanagawa.jp/ keikan/jorei/jorei.html (in Japanese).

13. Osgood, C. E., Suci, G. J. and Tannenbaum, P. H.; The Measurement of Meaning; University of Illinois Press, Urbana, IL (1957).

14. Nagamachi, M.; Kansei Engineering: A new ergonomic consumer-oriented technology for product development, Int. J. Ind. Ergonomics; Vol. 15, No. 1, pp. 3-12 (1995).

15. Jindo, T. and Hirasago, K.; Application studies to car interior of Kansei engineering, Int. J. Ind. Ergonomics; Vol. 19, No. 2, pp. 105-114 (1997).

16. Hsiao, S. W. and Huang, H. C.; A neural network based approach for product form design, Design Studies; Vol. 23, No. 1, pp. 67-84 (2002).

17. Kosaka, H. and Watanabe, K.; A Kansei product design system using neural network, Kansei Engineering International; Vol. 3, No. 4, pp. 31-36 (2003).

18. Kinoshita, Y., Cooper, E.W., Hoshino, Y. and Kamei, K.; Development of townscape evaluation system for colour planning support, Proc. 11th Int. Conf.

Human-Computer Interaction; No. 1279 (9 pages) in CD-ROM (2005).

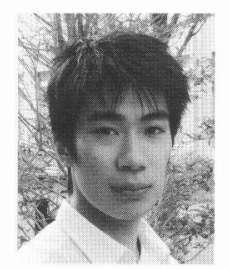

\section{Yuichiro KINOSHITA}

Yuichiro Kinoshita received the B.Eng. degree in computer science from Ritsumeikan University, Japan, in 2002. He is currently a Ph.D. student in the Department of Human and Computer Intelligence, Ritsumeikan University. His research interests include Kansei modelling, human factors, affective computing, and human-computer interaction. Mr. Kinoshita is a student member of the Japan Society of Kansei Engineering, the IEEE, ACM SIGCHI, and Human Interface Society in Japan.

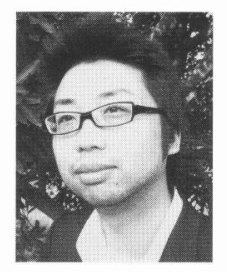

Yoshiaki SAKAKURA

Yoshiaki Sakakura received the B.Eng. and the Ph.D. degree in computer science from Ritsumeikan University, Japan, in 2001 and 2005, respectively. He is currently a Research Assistant in the Department of Human and Computer Intelligence, Ritsumeikan University. His research interests include optimization, personalized web search, and real world applications of evolutionary computation. Dr. Sakakura is a member of the IEEE, and Japan Society for Fuzzy Theory and Intelligent Informatics.

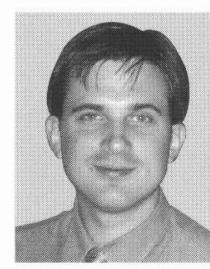

\section{Eric W. COOPER}

Eric W. Cooper received the B.A. degree in general fine arts from Maryland Institute College of Art, and the M.S. and the Ph.D. degree in computer science from Ritsumeikan University, Japan, in 1992, 1996 and 2002, respectively. He is an Associate Professor in the Department of Computer Science, Ritsumeikan University. His research is focused on color placement support systems. Other interests include color information processing, human interface design, and intelligent systems.

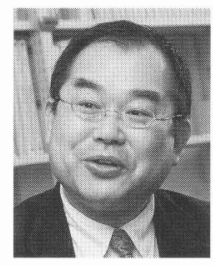

Katsuari KAMEI

Katsuari Kamei received the M.S. degree and the $\mathrm{Ph} . \mathrm{D}$. degree in electrical engineering from Ritsumeikan University, Kyoto, Japan, in 1980 and 1983, respectively. he is currently a Professor in the Department of Human and Computer Intelligence, Ritsumeikan University. His research interests include Kansei engineering, intelligent systems, human interfaces, and machine learning using soft computing technologies. 